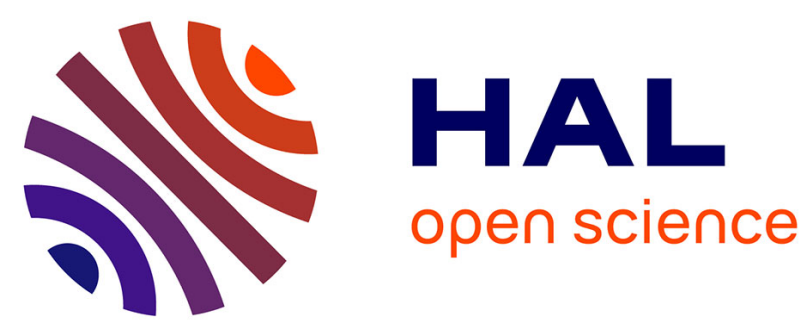

\title{
Antenna development for astroparticle and radioastronomy experiments
}

Didier Charrier

\section{To cite this version:}

Didier Charrier. Antenna development for astroparticle and radioastronomy experiments. 4th International workshop on Acoustic and Radio EeV Neutrino detection Activities - ARENA 2010, Jun 2010, Nantes, France. pp.S142-S146, 10.1016/j.nima.2010.10.141 . in2p3-00682534

HAL Id: in2p3-00682534 https://hal.in2p3.fr/in2p3-00682534

Submitted on 18 Dec 2014

HAL is a multi-disciplinary open access archive for the deposit and dissemination of scientific research documents, whether they are published or not. The documents may come from teaching and research institutions in France or abroad, or from public or private research centers.
L'archive ouverte pluridisciplinaire HAL, est destinée au dépôt et à la diffusion de documents scientifiques de niveau recherche, publiés ou non, émanant des établissements d'enseignement et de recherche français ou étrangers, des laboratoires publics ou privés. 


\title{
Antenna development for astroparticle and radioastronomy experiments
}

\author{
Didier Charrier, for the CODALEMA collaboration \\ Subatech, Ecole des Mines de Nantes - CNRS/IN2P3 - Université de Nantes, France
}

\begin{abstract}
An active dipole antenna is in operation since five years at the Nançay radio Observatory (France) in the CODALEMA experiment. A new version of this active antenna has been developed, whose shape gave its name of "Butterfly" antenna. Compared to the previous version, this new antenna has been designed to be more efficient at low frequencies, which could permit the detection of atmospheric showers at large distances. Despite a size of only $2 \times 1$ meters in each polarization, its sensitivity is excellent in the 30-80 MHz bandwidth. Three antennas in dual polarization were installed on the CODALEMA experiment, and four other have been recently installed on the Auger area in the scope of the AERA project. The main characteristics of the Butterfly antenna are detailed with an emphasis on its key features which make it a good candidate for the low frequency radioastronomy and the radio detection of transients induced by high energy cosmic rays.
\end{abstract}

Keywords: active antenna, LNA, fat dipole antenna, electrically short antenna, vector effective height

\section{Introduction}

Ultra high energy cosmic rays induce extensive air showers (EAS) and also an associated transient electric field. This can be detected in the so-called decametric range by an antenna whose main characteristics are a good sensitivity, a wide bandwidth (1-100 MHz), a high linearity to avoid intermodulation due to radio frequency interference (RFI) and also a linear phase response to maximize the amplitude of the received pulse. It has been demonstrated [1] that the optimum frequency range is the 5-85 MHz band but to avoid short waves and FM band RFI, the useful band is reduced to [25-85 MHz]. This is exactly the same frequency range required for the low frequency radio astronomy [2]. It means that if such an antenna sensitivity is high enough, ideally $10 \mathrm{~dB}$ lower than the galactic background, it should also work for the low frequency radio astronomy.

\section{Requirements to develop a wide bandwidth antenna}

To fulfill the wide band requirement, one can either design a passive or an active antenna. Log-Periodic Dipole Antennas (LPDA)[3] are passive antennas. They exhibit an almost constant radiation resistance and an almost null antenna reactance in their frequency ranges but their largest arms length are given by the lowest frequency, and consequently, they are necessarily huge for the $25-85 \mathrm{MHz}$ band. The $25 \mathrm{MHz}$ frequency would need a total arm length of $6 \mathrm{~m}$. To avoid building such a huge antenna, another possibility is to develop an active antenna. For that case, a dedicated Low Noise Amplifier (LNA) is placed close to the antenna radiator and consequently, a power matching is not needed which is a major advantage since the value of the LNA input impedance becomes a free parameter. It allows to develop an electrically short antenna and to use a very simple antenna radiator like a dipole. Indeed, the CODALEMA dipole antenna [4] and the Butterfly Antenna (Fig. 1) are both active fat dipole antennas with respectively a total arm length of $1.2 \mathrm{~m}$ and $2.2 \mathrm{~m}$.

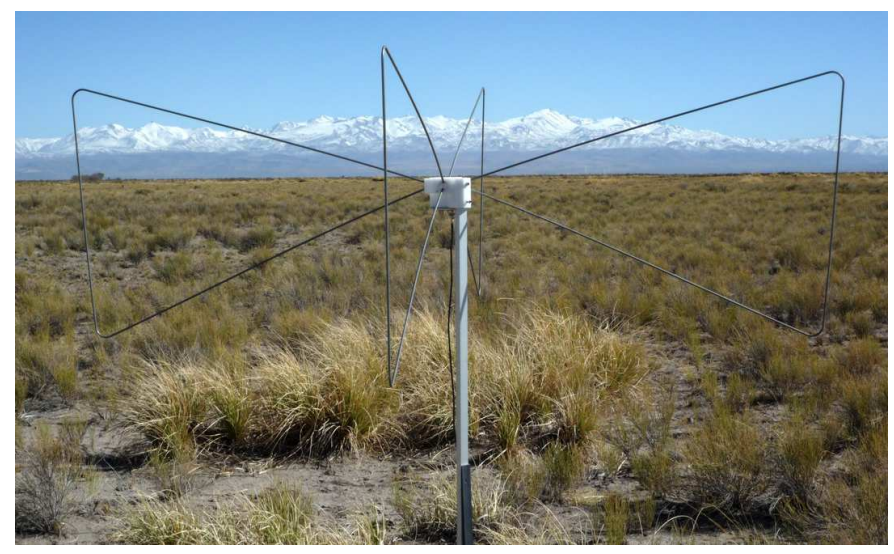

Figure 1: The Butterfly antenna, a dual polarization active antenna using an isosceles triangle as antenna radiator element, and a dual channel LNA located in a plastic mechanical frame placed at the antenna feedpoint.

\section{From the CODALEMA antenna to the Butterfly antenna}

An array of 24 CODALEMA dipole antennas is running on the field at Nançay for the detection of cosmic rays [5]. These antennas are linear single polarized and their simulated effective height ${ }^{1}$ is maximized at $100 \mathrm{MHz}$ for the zenith elevation.

\footnotetext{
${ }^{1}$ In the literature the effective height is defined as the ratio of the magnitude of the open-circuit voltage developed at the terminals of the antenna to the magnitude of the electric-field strength in the direction of the antenna polarization. It is not the definition that will be used here, see text.
} 
In this paper, the effective height is defined as the ratio of the magnitude of the voltage developed at the LNA input to the magnitude of the electric-field strength in the direction of the antenna polarization. The main motivation of an upgrade to the butterfly antenna is illustrated in Fig. 2. It represents the simulated values of the effective height for both antennas performed with the 4NEC2 software and calculated with MATLAB. For all the simulations of this paper, antenna losses are set to zero and the ground plane is assumed to be perfect. Note that the effective height has been improved for the 25-85 MHz band which is almost free of RFI in France, Argentina and many countries. The $25 \mathrm{MHz}$ sensitivity is $15 \mathrm{~dB}$ higher which should allow to enhance the detection of distant EAS. Moreover, RFI below $15 \mathrm{MHz}$ are cut in order to avoid the LNA being overdriven by them. Compared to the CODALEMA antenna, for the zenith elevation, FM band frequencies are attenuated. The second motivation for the Butterfly antenna is the detection of electric field with two horizontal linear polarizations, and the last one is the possibility to calibrate the antenna with the galactic background for the 30-80 MHz band.

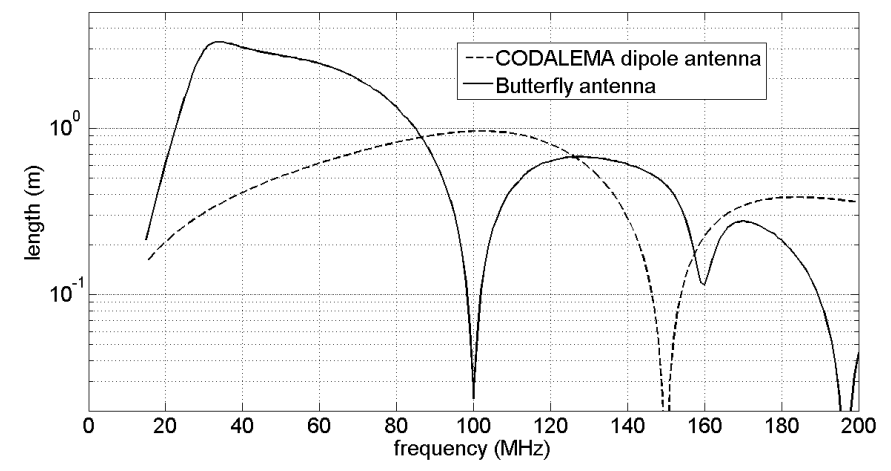

Figure 2: Effective heights of the CODALEMA antenna and the Butterfly antenna for the zenith elevation.

\section{Active antenna needs a dedicated input impedance LNA}

Considering $R_{\text {rad }}$ and $X_{\text {ant }}$ the antenna radiation resistance and antenna reactance, $R_{\operatorname{lna}}$ and $X_{\operatorname{lna}}$ the LNA input resistance and reactance, $\mathrm{G}$ the antenna gain, and assuming a lossless antenna, the magnitude of the antenna effective height $\mathrm{H}$ is:

$$
|H(f)|=\lambda \sqrt{\frac{G}{\pi Z_{0}}} \times \frac{\sqrt{R_{\mathrm{rad}}}\left|R_{\text {lna }}+j X_{\text {lna }}\right|}{\left|R_{\text {lna }}+j X_{\text {lna }}+R_{\text {rad }}+j X_{\text {ant }}\right|}
$$

with $Z_{0}$ the intrinsic impedance and $\lambda$ the wave length of the received electric field. From 1, we deduce that the effective height is maximized if $X_{\text {lna }}$ is cancelling $X_{\text {ant }}$, if $R_{\text {rad }}$ is maximized and if $R_{\text {lna }}$ is much greater than $R_{\text {rad }}$. We also deduce that the effective height is flatter if $R_{\text {rad }}$ variation is minimized. The cancellation of $X_{\text {ant }}$ by $X_{\text {lna }}$, as illustrated in Fig. 4 is partly obtained by adding a shunt inductance in parallel with the LNA input. This cancellation is more easily obtained if $X_{\text {ant }}$ variation is minimized. We also observe on Fig. 3 that, with this inductance, $R_{\text {lna }}$ becomes greater than $R_{\text {rad }}$ on a wider bandwith. Another advantage of this inductance is to cut low frequencies RFI.
For a dipole antenna, the minimization of $R_{\text {rad }}$ and $X_{\text {ant }}$ variations is obtained by increasing the diameter of the dipole [6], thus becoming a fat dipole. Indeed, if we modelize to the first order the antenna impedance by a serial L-C resonant circuit, for a given resonance frequency depending on the overall antenna length, the greater the antenna capacitance and the lower the Q-factor. So the dipole antenna broadband capabilities are increased by its capacitance value.
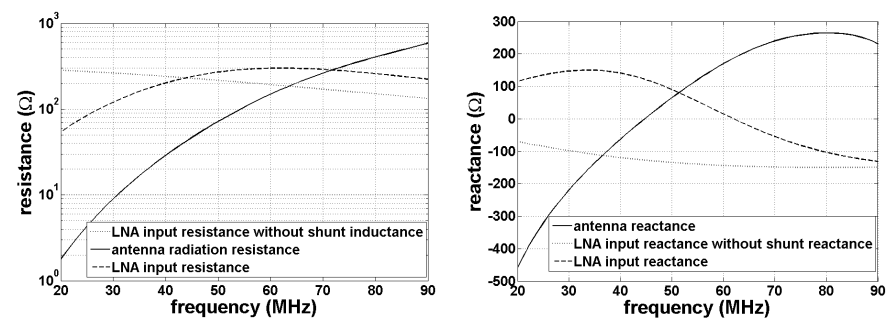

Figure 3: antenna radiation resistance and LNA input resistance.

Figure 4: antenna reactance and LNA input reactance.

\section{Sizing the Butterfly antenna radiator}

The shape of one arm of the Butterfly antenna (Fig. 1) is an isosceles triangle made of an aluminium rod with a diameter of $6 \mathrm{~mm}$. This simple shape is chosen since it is a tradeoff between mechanical complexity, radiator weight, wind robustness, cost, and the antenna capacitance. The shape could have been a rectangle finished by a triangle on the antenna terminal side, but the advantage of a triangle is that only two bends are required to build one arm. The important parameters are the length of the outline which gives the resonance frequency and the fact that the radiator shape is equivalent to a fat rod, increasing the broadband capabilities [6]. Finally, with a triangle-shaped radiator, seen from the terminal, the two arms are ended like a point, it reduces the parasitic shunt capacitance [7]. This antenna radiator can be seen as a slice of a cone whose broadband characteristic would be better but quite impossible to built. Indeed, a 4NEC2 simulation gives a capacitance of $12 \mathrm{pF}$ when it would have been twice more with a cone. Nevertheless, the obtained capacitance is higher than the one it would have been for a simple thin dipole $(7 \mathrm{pF})$. A resonance frequency of roughly $50 \mathrm{MHz}$ was chosen since it is the middle of the $20-80 \mathrm{MHz}$ band. Consequently, at this frequency, the current distribution along the antenna radiator is a half sine period. It means that the half perimeter of one triangular arm must be one-quarter wavelength, which gives $1.5 \mathrm{~m}$. The Butterfly antenna can be considered as a wire bow-tie antenna only for frequencies above its first resonance frequency. It has been demonstrated [6] that for this kind of antenna a flare angle ranging from $30^{\circ}$ to $50^{\circ}$ is optimum to increase its broadband characteristics. If we choose a flare angle of $45^{\circ}$, with a perimeter of $3 \mathrm{~m}$, we deduce that the triangular arm has a base of $0.84 \mathrm{~m}$ and a height of $1 \mathrm{~m}$. This is a good compromise since it forms an antenna with an overall length of roughly $2 \mathrm{~m}$. But for mechanical constraint, in the case of the Butterfly the base has been reduced to $0.75 \mathrm{~m}$ and the height thus raises to $1.07 \mathrm{~m}$. 


\section{The Low Noise Amplifier}

The LNA itself, named CODALAMP, is an ASIC [4] designed at SUBATECH in 2004, using the AMS BiCMOS $0.8 \mu \mathrm{m}$ technology. A specific two-channel LNA board using two of these ASICs has been developed recently for the Butterfly antenna. The LNA board input impedance is equivalent to a resistance of $300 \Omega$ in parallel with an input capacitance of $6 \mathrm{pF}$. An external $1 \mu \mathrm{H}$ inductance is added in parallel on the input. The LNA board voltage gain, defined as the ratio of the output voltage on a $50 \Omega$ terminal load over the voltage on the input impedance, is $26 \mathrm{~dB}$; the bandwidth is more than $200 \mathrm{MHz}$; the input $1 \mathrm{~dB}$ compression point is $25 \mathrm{mV}$ peak to peak (p-p); the output reflection coefficient is better than $-20 \mathrm{~dB}$ in the 4$210 \mathrm{MHz}$ band; the gain drift with temperature is $-0.026 \mathrm{~dB} / \mathrm{K}$ and the total consumption is $104 \mathrm{~mA}$ under a voltage between 6 and $15 \mathrm{~V}$. The two analog outputs use N-type connectors and the power supply is fed by the output signal wire.

\section{Response of the Butterfly antenna to the galactic back- ground}

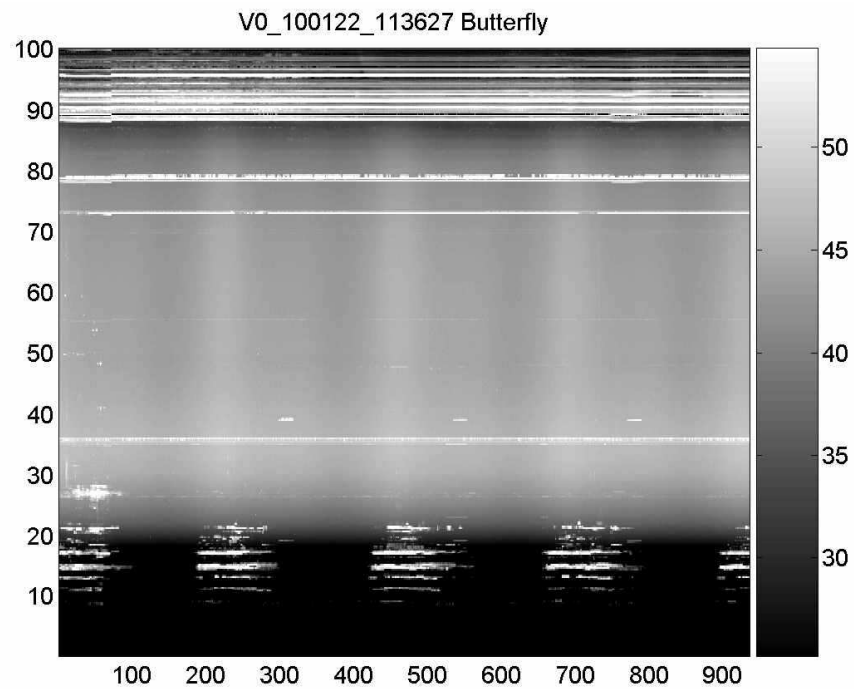

Figure 5: Measurement of the galactic drift over 4 days with the Butterfly antenna. $\mathrm{Y}$-axis is the frequency in $\mathrm{MHz} ; \mathrm{X}$-axis is the time, same scale as Fig 6.

Using the galactic temperature as a calibrating signal is very convenient to measure the active antenna sensitivity and to evaluate the active antenna modelization with respect to calculations. The antenna was placed on a test bench at the Nançay radio observatory, in the East-West polarization. It consists in acquiring continuously spectra with a spectrum analyser during a few days in order to reveal the galactic drift. For this measurement a sharp 24-82 MHz filter is placed on the front of the spectrum analyser to avoid its intermodulation. In Fig 5 there are no traces of third order intermodulation above the short waves band or below the FM band, where they should have appeared if any: it means that the LNA linearity is large enough. Moreover in Fig. 6 which represents a slice of Fig. 5 for the $55 \mathrm{MHz}$ frequency, we observe a galactic drift of $2.8 \mathrm{~dB}$. In Fig. 6 is also

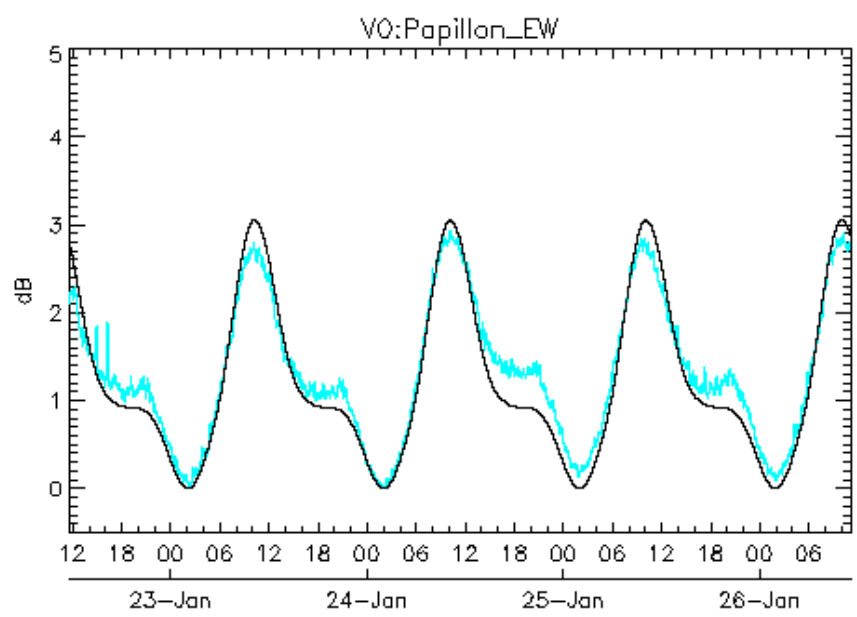

Figure 6: Simulation(black line) versus measurement(cyan line) of the galactic noise drift at $55 \mathrm{MHz}$ over 4 days. Y-axis is a relative noise density level in decibel, $X$-axis is the UTC time.

plotted a simulation of the $55 \mathrm{MHz}$ galactic drift performed by the projection of the Butterfly antenna gain on a map of the sky temperature generated with the LFmap code [8]. It gives a theoretical drift of $3 \mathrm{~dB}$ which is very close to the $2.8 \mathrm{~dB}$ measured. This means that the galactic noise dominates the LNA noise at $55 \mathrm{MHz}$. In Fig. 6 we also observe that the shape of the measured galactic drift follows well the simulation. On the edges of the bandwidth, we still measure a drift of $2.5 \mathrm{~dB}$ and $2.7 \mathrm{~dB}$ at 30 and $80 \mathrm{MHz}$. Consequently, the Butterfly antenna could be a good candidate as an element of a big array for the low frequency radio astronomy [2].

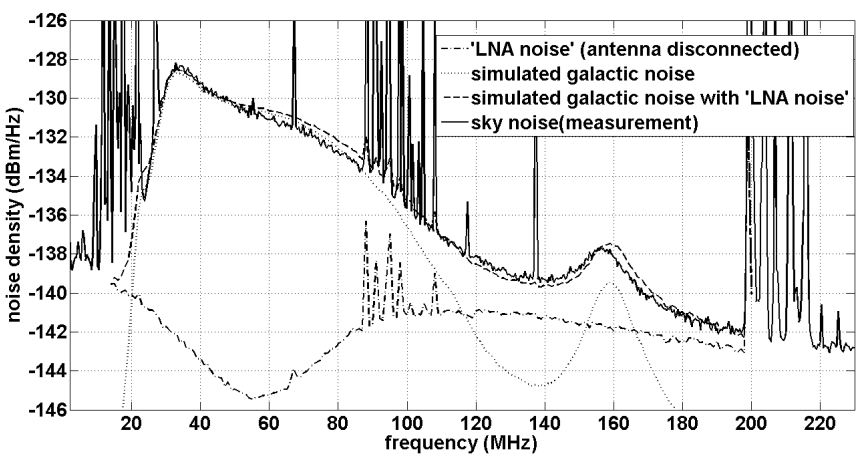

Figure 7: Simulation(top dashed line) versus measurement(top full line) of the absolute galactic noise level performed at the output of the LNA board. The total simulated noise includes the measured LNA noise.

We also observe in Fig. 7 that the galactic response is quite flat in the $25-85 \mathrm{MHz}$ band; the variation of $-6 \mathrm{~dB}$ between 33 and $88 \mathrm{MHz}$ is due to the drop out of the galactic temperature with the frequency. The symmetry of the antenna is good, since the two polarizations received almost the same level of the galactic background. The Fig. 7 is a comparison between a measurement of the galactic background realized at the Pierre Auger observatory in Argentina and its calculation. This calculation is performed with known data of the minimum galactic temperature, the simulated antenna impedance and the mea- 
sured values of LNA S-parameters; it gives the absolute value of the foreseen galactic noise without any adjustments. We observe an error between measurement and calculation lower than $1 \mathrm{~dB}$ from 25 to $200 \mathrm{MHz}$, meaning that the simulated value of the antenna impedance and the MATLAB algorithm used to calculate the galactic background are reliable. In Fig. 7, the LNA noise is measured by disconnecting the antenna radiator, this cannot thus be an exact value of the LNA noise since it depends on the antenna impedance.

\section{Butterfly antenna transient response}

The modelization of the magnitude response is important, but is not enough from the transient detection point of view. The phase response modelization is also needed in order to deconvolute the transient output signal from the vector antenna response and then, calculate the shape and the magnitude of the received electric field. This work is performed in the frequency domain with the 4NEC2 software. The vector values of the electric field generated by 4 NEC 2 are used to calculate the antenna vector effective height, then the input electric field is simply obtained by a deconvolution. Rather than plotting the antenna directivity [6] for many frequencies it is more clever to plot the active antenna response to a Dirac for many polar and azimuth angles: this represents a particular feature of the antenna transfer function itself. The value of the maximum amplitude could be used to define a kind of time domain directivity. This "time directivity" could be generalized to different kinds of received electric pulses and then parameterized by raising and falling time of the input pulse, which depend on the distance of the EAS. In Fig 8 is plotted the Butterfly antenna response to a Dirac input electric field for a few polar angles, from the zenith to $80^{\circ}$, and for the E-plane [6]. We observe that the shape is slightly elevationdependent and that the amplitude is changing by a factor 2 up to $60^{\circ}$. For the H-plane the amplitude changes only by a factor 2 from the zenith to $80^{\circ}$.

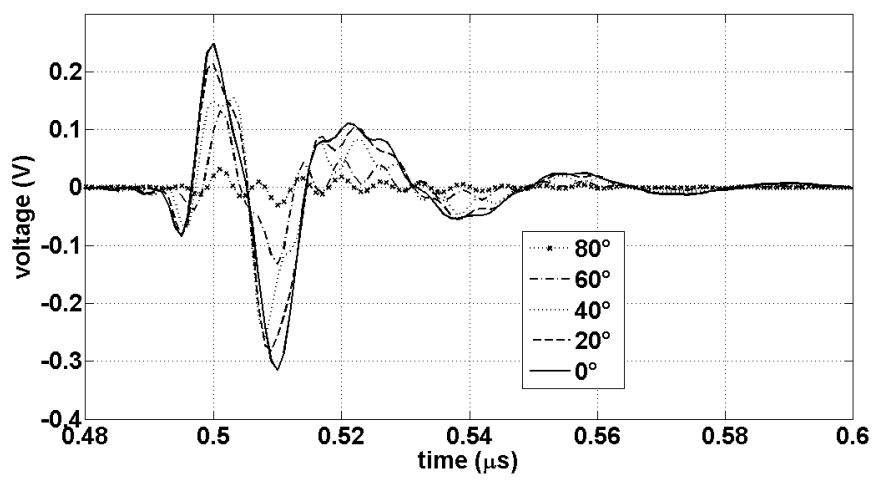

Figure 8: The E-plane Butterfly antenna response to a Dirac electric field transient parametrized by the polar angle ranging from zenith to $80^{\circ}$.

The Fig. 9, top, represents a pulse generated by a high energy cosmic ray coming from the zenith, detected by a Butterfly antenna and acquired by an autonomous, self-triggered station [9] of the CODALEMA experiment. This pulse is numerically filtered within a 20-87 MHz band to suppress RFI. We observe that this signal is similar to the simulated active antenna response to a Dirac pulse, see Fig. 8. The difference is due to the fact that the received electric field is not exactly a Dirac pulse. After a deconvolution of this pulse by the active antenna response, we obtain, for the $20-87 \mathrm{MHz}$ band, the electric field received by the antenna as illustrated in Fig. 9, bottom. It can be clearly concluded that the electric field generated by an EAS (at least, for this example) is a short pulse. This is a very preliminary work since this first result is based only on one event, so the shape of induced electric field will have to be investigated with more statistics and more accuracy, for example in order to answer the question whether it is bipolar.

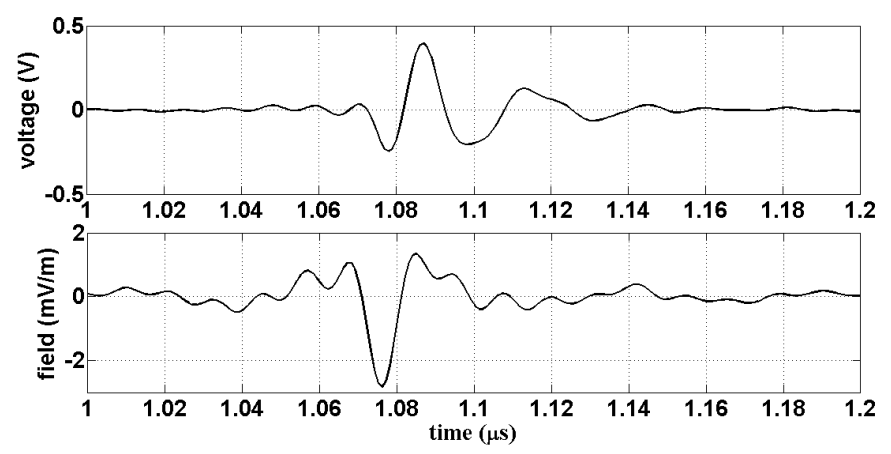

Figure 9: Top, an event acquired by the autonomous station [9]. Bottom, the induced electric field generated from the deconvolution of the top curve by the antenna vector transfer function.

\section{Conclusion and outlook}

Active antennas allow to develop both compact and sensitive antennas for the $25-85 \mathrm{MHz}$ bandwidth. With the Butterfly antenna, good agreement between measurements and simulations are obtained for the galactic background drift, for the absolute galactic temperature and for the impulse antenna response. The very good sensitivity of the Butterfly antenna to the galaxy should allow calibrating it. The modelization of the vector effective height allows knowing accurately the shape and the value of the induced electric field. Due to its sensitivity and compactness, the Butterfly antenna is as well a good candidate for low frequency radio astronomy [2]. A mass production of Butterfly antennas will be performed before end of 2010, at least for the extension of the CODALEMA experiment.

\section{References}

[1] C. Ruehle, for the Pierre Auger Collaboration, this issue.

[2] J. Girard, P. Zarka, D. Charrier, L. Denis, A. Konovalenko, M. Tagger and C. Tasse, Planetary Radio Emissions VII, Graz, Austria, 15-17/9/2010.

[3] O. Seeger, for the Pierre Auger Collaboration, this issue.

[4] D. Charrier, for the CODALEMA collaboration, in: APS International Symposium, IEEE, 2007, 10.1109/APS.2007.4396539.

[5] D. Ardouin et al., Astroparticle Physics 31 (2009) 192.

[6] C.A. Balanis, Antenna Theory- 3rd Edition, Wiley.

[7] J.D. Kraus, Antennas, McGraw-Hill, New York, 1988.

[8] <www.ece.vt.edu/swe/lwa/memo/lwa0111.pdf>.

[9] T. Garçon, for the CODALEMA collaboration, this issue. 